\title{
Automated differentiation of Alzheimer's condition using Kernel Density Estimation based texture analysis of single slice brain MR images
}

\begin{abstract}
Computer-assisted tools can aid in the detection of Alzheimer disease (AD) which is a progressive neurodegenerative disorder that can lead to cognitive impairments and eventually death. The accumulated effects due to $\mathrm{AD}$ can cause changes in the appearance of grey matter, white matter and cerebrospinal fluid in brain Magnetic Resonance (MR) images. This study aims to use Kernel Density Estimation (KDE) technique to analyse the textural changes from single slice brain MR images for the detection of AD. The preprocessed, skull stripped T1-weighted MR brain images are obtained from the publicly available OASIS database. A single axial slice per subject is chosen from a volumetric image for further processing to reduce the computational load. Multivariate KDE technique is applied to each pixel, by considering the changes in the neighbourhood based on selected bandwidth to obtain corresponding density estimates. Statistical features quantifying the distribution of density estimates are extracted to characterise textural variations in images. Linear discriminant analysis (LDA) classifier is implemented with ten-fold cross-validation for detecting AD. An optimum bandwidth of 18 for the KDE technique is selected based on the classification performance. Out of seven extracted texture features, three are found to be statistically significant in distinguishing AD. The
\end{abstract}

\footnotetext{
*Corresponding author: Amtutha Veluppal: Department of Applied Mechanics, Indian Institute of Technology Madras, Chennai, India, amrutha.qwerty@gmail.com

Deboleena Sadhukan Department of Applied Mechanics, Indian Institute of Technology Madras, Chennai, India, deboleena.rainbow@gmail.com

Venugopal Gopinath Department of instrumentation and Control Engineering, N.S.S. College of Engineering, Palakkad, India, venugopalg@gmail.com

Ramakrishnan Swaminathan Department of Applied Mechanics, Indian Institute of Technology Madras, Chennai, India, sramki@iitm.ac.in
}

classification with LDA yields an accuracy of $72.3 \%$ with a sensitivity of $80.6 \%$ for identifying $\mathrm{AD}$ from healthy subjects. The proposed method is efficient in detecting AD by revealing the textural changes within the brain slice without the involvement of any segmentation technique. Thus, the novel KDE-based texture analysis proves to be an effective tool for the automated diagnosis of $\mathrm{AD}$ from single slice brain $\mathrm{MR}$ images.

Keywords: Alzheimer disease, Kernel density estimation, texture analysis, 2D brain images, Linear discriminant analysis.

https://doi.org/10.1515/cdbme-2021-2191

\section{Introduction}

Alzheimer's disease is the most common form of dementia, accounting for nearly $70 \%$ of all cases. It is a progressive neurodegenerative disorder that is increasingly becoming a lethal and burdening disease in the ageing world population [1]. It is characterised by symptoms such as deterioration in memory and cognitive skills, behavioural deficits, and inability to perform daily activities as the severity increases. $\mathrm{AD}$ is histologically characterised by deposition of plaques and formation of neurofibrillary tangles, which can lead to a cascade of degeneration in brain cells, leading to atrophy patterns across brain [2].

In recent decades neuroimaging techniques are becoming a popular non-invasive way of detecting $\mathrm{AD}$. The most commonly used techniques are - computed tomography (CT), Magnetic Resonance Imaging (MRI) and Positron Emission Tomography (PET) imaging modalities. MRI is most prevalent among these techniques due to its high tissue contrast, spatial resolution, low cost, and widespread availability [3] 
Researchers are focusing on developing computerassisted tools in identifying $\mathrm{AD}$ to streamline the detection process and reduce the health care burden. Voxel-based morphometry requires handling and processing huge amounts of data [4]. Region Of Interest (ROI) based approaches require identification of a particular patch or structure in the brain, from which features are extracted. However, these studies require expert knowledge to identify informative ROI from the brain [5]. The whole-brain approach provides the advantage of not requiring prior knowledge or involvement of segmentation techniques for delineating particular structures for analysis. Elshatoury et al. proposed histogram-based analysis with majority voting on multiple slices of brain images in sagittal plane for detecting $\mathrm{AD}$ [6]. Alam et al. used dual-tree complex wavelet transform and the principal component analysis for analysing morphometric changes on manually extracted transaxial slices to identify AD subjects [7]. A whole-brain multimodal MRI and PET-based deep learning model is proposed in [8]. However, analysis of the whole brain requires handling large amount of data which is computationally intensive. Single slice criterion analysis can provide insight into coarse changes occurring across multiple structures with the additional advantage of reduced complexity [5].

Texture based analysis has gained attention in recent years as it can capture complementary information other than the ones provided by volume or shape features [9]. The conventional image texture analysis approaches often involve statistical techniques such as grey-level co-occurrence matrix and grey-level run-length matrix, which analyses the distribution and relationship among the grey-level pixel intensities [10]. These methods often involve the computation of a large number of features, as the pixel relations are considered for various angles and directions [11].

Kernel Density Estimation (KDE) is a nonparametric technique for the estimation of probability densities of random variables [12]. Spatial KDE can be applied to images to obtain the density estimates, which captures the local variations in the pixel intensities. Since the obtained density estimates at each pixel location signify the probability density of the corresponding intensity values based on its neighbourhood pixels, it can be treated as a texture analysis tool. The KDE values can prominently represent the local textural changes.

The main objective of this study was to characterise the pattern variations in brain tissues from single slice brain Magnetic Resonance (MR) images using novel KDE-based texture analysis to differentiate normal and AD subjects. The computed texture features show a distinction between normal and AD subjects. The Linear Discriminant Analysis (LDA) model is applied to distinguish diseased condition using the proposed texture analysis technique.

\section{Methodology}

\subsection{Image Database}

Images used in this study consists of T1-weighted Magnetic Resonance (MR) transaxial brain images of 92 normal and 92 AD subjects obtained from publicly available Open Access Series of Imaging Studies (OASIS) cross-sectional database [13]. The demographics of the subjects are shown in Table.1. The normal and $\mathrm{AD}$ subjects are divided based on Clinical Dementia Rating (CDR) scores. The $90^{\text {th }}$ transaxial slice from skull stripped brain volume is considered for further analysis.

Table 1: Demographic record of subjects.

\begin{tabular}{lll}
\hline Class & CDR & Age (Mean \pm SD) \\
\hline Normal & 0 & $68.11 \pm 5.43$ \\
AD & $0.5,1$ & $76.67 \pm 7.06$ \\
\hline
\end{tabular}

\subsection{Feature extraction}

The pipeline to differentiate normal and $\mathrm{AD}$ images using KDE based texture analysis of single slice brain MR images is shown in Figure.1. All the images were cropped to fit the brain, which resulted in a dimension of $167 \times 137$.

$\mathrm{KDE}$ technique is applied to the images, and corresponding density estimates are evaluated for each pixel. Spatial multivariate kernel density estimation can be applied to $2 \mathrm{D}$ images. Here, the pixel intensities and the spatial coordinates are considered as variables used to calculate the density estimates of each pixel [14]. KDE technique estimates the distribution of a finite set of pixels from a neighbourhood by placing a kernel function of a fixed bandwidth at each points and taking the sum of all kernels [12].

The Gaussian kernel is employed for KDE technique in this study. For pixel intensities $x_{1}, x_{2}, x_{3} \ldots x_{N}$ in a 2D image, the probability density estimate $\mathrm{f}(x)$ at each point $x$ can be calculated as follows [14]

$$
f\left(x_{i}\right)=\frac{1}{N B} \sum_{i=1}^{N} \prod_{j=1}^{d} \mathrm{~K}\left(\frac{x_{j}-x_{i j}}{B}\right)
$$

where, $x_{i j}$ is the $j^{\text {th }}$ dimension of the $i^{\text {th }}$ pixel and $x_{j}$ is the $j^{\text {th }}$ dimension of $x . K$ represents radial isotropic Gaussian kernel function and $B$ is the bandwidth that determines the size of the kernel. 


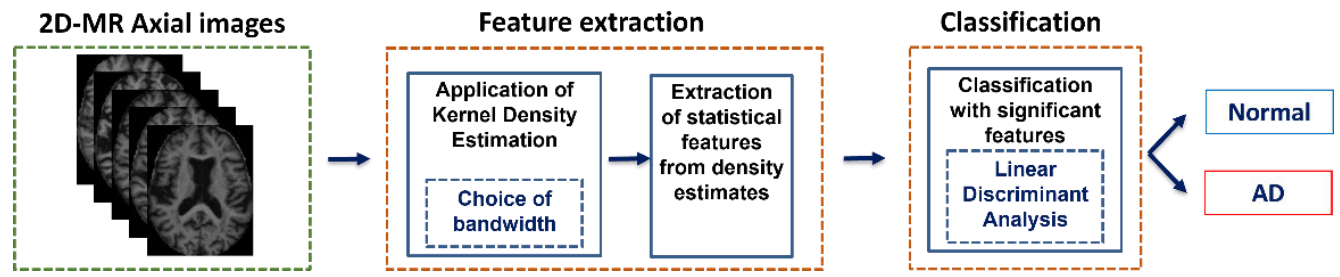

Figure 1: Block diagram of the proposed methodology

In $\mathrm{AD}$, the atrophy from multiple structures can alter the textural pattern in brain images due to shrinkage in grey and white matter tissue. KDE encodes the distribution of image pixels and characterises the underlying textural patterns of the image. The optimum bandwidth for the current application is selected based on classification accuracy by varying the bandwidth. The statistical features such as mean, median, variance, kurtosis, skewness, and entropy are extracted from the density estimates of each image. The significant features are selected based on Wilcoxon rank-sum test. Further, Linear Discriminant Analysis (LDA) classifier is employed with 10fold cross-validation technique to classify normal and $\mathrm{AD}$ images. LDA is advantageous for having a low computational cost.

\section{Results}

The representative brain MR images of normal and $\mathrm{AD}$ subjects are shown in Figure.2 (a) and (b), respectively. It is observed that there is shrinkage in white matter and grey matter tissues, leading to increased cerebrospinal fluid-filled areas such as centrally located lateral ventricles in $\mathrm{AD}$ subjects.

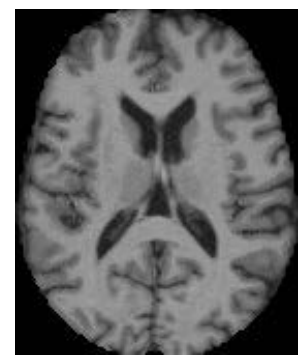

(a)

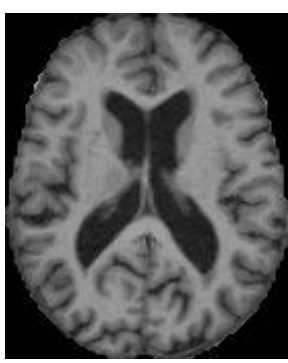

(b)
Figure 2: Representative MR brain images in the axial view of (a) Normal and (b) AD subjects

The optimum bandwidth for KDE technique to suitably capture the textural variations is selected based on classification performance. For this, the bandwidth is varied from 4 to 20 and accuracy is evaluated. Figure. 3 depicts the variation in classification accuracy with bandwidth for differentiating normal and AD groups.

A narrow bandwidth may capture random pixel fluctuations, whereas a large bandwidth may overlook the local variations [15]. Thus, from the figure, it is seen that an optimum bandwidth of 18 achieves the highest accuracy and therefore selected for the current application.

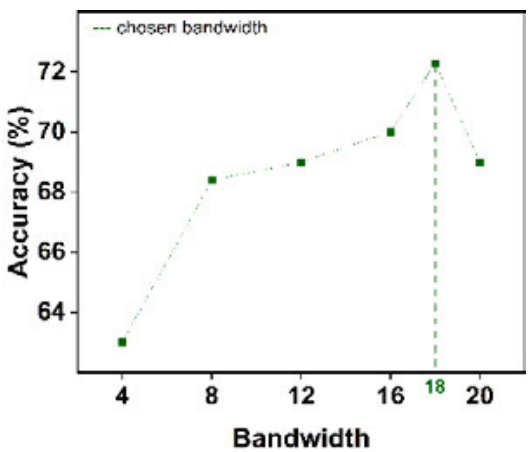

Figure 3: Variations in classification accuracy with bandwidths in differentiating Normal and AD groups

Figure. 4 demonstrates the variations in features between normal and $\mathrm{AD}$ subjects. Among the extracted features variance, kurtosis and entropy are found to be significant with $p<0.05$. The entropy and variance are observed to be high for normal images compared to AD. This infers that the spread of pixel intensities is less for the abnormal condition. This may be due to the presence of enlarged ventricles and widespread loss of brain tissues observed in AD. Kurtosis describes whether the distribution of density estimates is more spreadout or peaked around mean. It is observed to be higher for AD subjects.

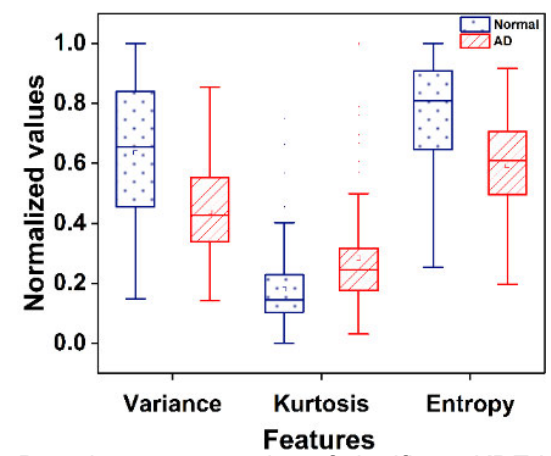

Figure 4: Box plot representation of significant KDE based texture features 
Further, an LDA classifier is employed for differentiating normal and $\mathrm{AD}$ groups with the proposed KDE based texture features. The classification performance of the LDA model with these features yields a sensitivity, specificity and accuracy of $80.6 \%, 68 \%$ and $72.3 \%$, respectively.

\section{Conclusion}

Automated detection tools with MR images can aid in the rapid clinical diagnosis of $\mathrm{AD}$. The kernel density estimation technique is able to characterise the textural variations from images by incorporating both intensity and spatial information. In this study, KDE-based features are employed to capture the textural pattern changes in transaxial brain images to distinguish normal and AD subjects. The proposed methodology achieves reasonably high accuracy in detecting $\mathrm{AD}$ with only three features. The use of a single slice eliminates the need for segmentation techniques to extract ROI. Thus, this pipeline can be used for automated mass screening of $\mathrm{AD}$ patients with minimum medical expertise.

\section{Author Statement}

Research funding: The author state no funding involved. Conflict of interest: Authors state no conflict of interest. Informed consent: Informed consent has been obtained from all individuals included in this study. Ethical approval: The research related to human use complies with all the relevant national regulations, institutional policies and was performed in accordance with the tenets of the Helsinki Declaration, and has been approved by the authors' institutional review board or equivalent committee.

\section{References}

[1] Patterson C. World Alzheimer report 2018. 2018.

[2] Mendoza-Léon R, Puentes J, Uriza LF, Hoyos MH. Singleslice Alzheimer's disease classification and disease regional analysis with Supervised Switching Autoencoders. Comput. Biol. Med. 2020;116:103527.

[3] Zhao X, Ang CKE, Acharya UR, Cheong KH. Application of Artificial Intelligence techniques for the detection of Alzheimer's disease using structural MRI images. Biocybern Biomed Eng.2021.

[4] Schmitter D, Roche A, Maréchal B, Ribes D, Abdulkadir A, Bach-Cuadra $M$, et al. An evaluation of volume-based morphometry for prediction of mild cognitive impairment and Alzheimer's disease. Neurolmage Clin. 2015;7:7-17.

[5] Islam J, Zhang Y. Brain MRI analysis for Alzheimer's disease diagnosis using an ensemble system of deep convolutional neural networks. Brain Inform. 2018;5(2):1-14.

[6] Elshatoury H, Avots E, Anbarjafari G, ADNI. Volumetric histogram-based Alzheimer's disease detection using support vector machine. J Alzheimers Dis. 2019;72(2):515-24.

[7] Alam S, Kwon GR, Kim Jl, Park CS. Twin SVM-based classification of Alzheimer's disease using complex dual-tree wavelet principal coefficients and LDA. J. Healthc. Eng. 2017;2017.

[8] Liu M, Cheng D, Wang K, Wang Y. Multi-modality cascaded convolutional neural networks for Alzheimer's disease diagnosis. Neuroinformatics. 2018;16(3):295-308.

[9] Chincarini A, Bosco P, Calvini P, Gemme G, Esposito M, Olivieri C, et al. Local MRI analysis approach in the diagnosis of early and prodromal Alzheimer's disease. Neuroimage. 2011;58(2):469-80.

[10] Kassner A, Thornhill R. Texture analysis: a review of neurologic MR imaging applications. AJNR Am. J. Neuroradiol. 2010;31(5):809-16.

[11] Cai JH, He Y, Zhong XL, Lei H, Wang F, Luo GH, et al. Magnetic resonance texture analysis in Alzheimer's disease. Acad. Radiol. 2020;27(12):1774-83.

[12] Scott DW. Multivariate density estimation: theory, practice, and visualisation: John Wiley \& Sons; 2015.

[13] Marcus DS, Wang TH, Parker J, Csernansky JG, Morris JC, Buckner RL. Open Access Series of Imaging Studies (OASIS): cross-sectional MRI data in young, middle-aged, nondemented, and demented older adults. J. Cogn. Neurosci. 2007;19(9):1498-507.

[14] Xie CH, Yang XW, Liu Z, Liu YJ, Chang JY. First-order kernel density estimation of abdomen medical image intensity and spatial information and application to segmentation. Optik. 2014;125(22):6648-56.

[15] Economou G, Fotinos A, Makrogiannis S, Fotopoulos S, editors. Color image edge detection based on nonparametric density estimation. Proceedings 2001 International Conference on Image Processing (Cat No 01CH37205); IEEE. 2001;1:922-925. 